\title{
Magnetic Near Field Imaging with Electron Energy Loss Spectroscopy Based on Babinet's Principle
}

Michal Horák ${ }^{1}$, Vlastimil Křápek ${ }^{2}$, Martin Hrtoň ${ }^{1}$, Andrea Konečná ${ }^{3}$, Filip Ligmajer ${ }^{2}$, Michael StögerPollach $^{4}$ and Tomáš Šikola ${ }^{2}$

${ }^{1}$ CEITEC Brno University of Technology, Brno, Jihomoravsky kraj, Czech Republic, ${ }^{2}$ CEITEC Brno University of Technology and Institute of Physical Engineering, Brno University of Technology, Brno, Jihomoravsky kraj, Czech Republic, ${ }^{3}$ Materials Physics Center CSIC-UPV/EHU, San Sebastian, Navarra, Spain, ${ }^{4}$ USTEM TU Wien, Wien, Wien, Austria

Localized surface plasmon resonances (LSPR) are self-sustained collective oscillations of free electrons in metal nano- and microstructures, often called plasmonic antennas. LSPR can be characterized by scanning transmission electron microscopy (STEM) combined with electron energy loss spectroscopy (EELS) which utilizes an electron beam that interacts with the plasmonic antenna and excites the LSPR. EELS measures the energy transferred from electrons to the LSPR and it is sensitive to the electric near field of LSPR [1].

Babinet's principle, originating in the wave theory of light and diffraction, relates the properties of a planar plasmonic antenna (particle) and a complementary aperture in a thin metal film of the same size and shape [2]. In particular, the energies of LSPR in both antennas shall be identical and the electric/magnetic field distribution of the solid antenna shall correspond to the magnetic/electric field distribution of the complementary aperture. This link can be readily used to study the magnetic near field by measuring the electric near field by EELS in the complementary structure.

We present a study of Babinet's principle of complementarity in plasmonics focused on measuring the magnetic near field by EELS. We have studied a set of gold plasmonic antennas fabricated in the form of particles and apertures in a gold layer by focused ion beam lithography, which is a suitable technique for fabrication of a small series of plasmonic antennas [3] allowing to fabricate easily both types of antennas (particles and apertures) on the same sample. We have studied bow-tie and diabolo antennas [4] and elementary disc-shaped plasmonic antennas [5].

The first part of our study deals with plasmonic antennas featuring areas of extremely concentrated electric or magnetic field, known as hot spots. We combined two types of electric-magnetic complementarity to increase the degree of freedom for the design of the antennas: bow-tie and diabolo duality and Babinet's principle. The role of Babinet's principle in interchanging electric and magnetic field hot spots and its consequences for practical antenna design are discussed. We have revisited plasmonic modes in nanoparticle dimers with conductive (diabolo or inverted bow-tie antennas) or insulating (bow-tie or inverted diabolo antennas) junction. In our study combining EELS, optical spectroscopy, and numerical simulations we show coexistence of strongly and weakly hybridized modes [4]. We show that Babinet's principle allows engineering the near field of plasmonic modes independent of their energy. Further, we show that combined EELS imaging of a plasmonic antenna and its Babinet-complementary counterpart allows to reconstruct the distribution of both electric and magnetic near fields of LSPR supported by the antenna as well as charge and current antinodes of related charge oscillations.

We have to do the following steps to map the electric and magnetic near field of a LSPR mode in the plasmonic antenna: First, we have to record an EELS spectrum image of the particle antenna and the corresponding aperture. Second, we have to extract the EEL spectrum and identify the energy of a specific 
LSPR mode as illustrated in Figure 1 (a). Third, we have to extract the loss probability maps at the energy of a specific LSPR from both spectrum images so we obtain the results shown in Figure 1(b) for the longitudinal dipole mode in a diabolo antenna. The loss probability map at the LSPR energy for the particle antenna represents the out-of-plane electric near field distribution of the mode in the particle and the loss probability map recorded for the complementary aperture represents the out-of-plane magnetic near field distribution of the mode in the particle. Note that the qualitative correspondence between the simulated near field distributions in Figure 1(c) and measured loss probability maps in Figure 1(b) is very good. Consequently, this strategy allows us to detect the magnetic field hot spot in a diabolo antenna.

The second part of our study deals with disc-shaped plasmonic antennas. We have confirmed the qualitative validity of Babinet's principle, but we have found some quantitative differences. By comparing the experimental data with a theoretical model we found that differences originate both from the limited theoretical validity of the Babinet's principle. In particular, apertures were found to exhibit stronger plasmonic response than solid antennas, which makes them a remarkable alternative of the usual plasmonic antennas design [5]. Figure 2 shows the mapping of the LSPR in a gold disc-shaped plasmonic particle and aperture.

To conclude, we have demonstrated a possibility of the magnetic field imaging with EELS based on Babinet's principle. We have studied gold plasmonic antennas with electric and magnetic hot spots demonstrating a possibility to qualitatively visualize the magnetic field including the magnetic hot spot by EELS. Nevertheless, a detailed analysis for disc-shaped antennas revealed quantitative limits in the validity of Babinet's principle, rendering the magnetic field imaging based on the Babinet's principle only qualitative [6].

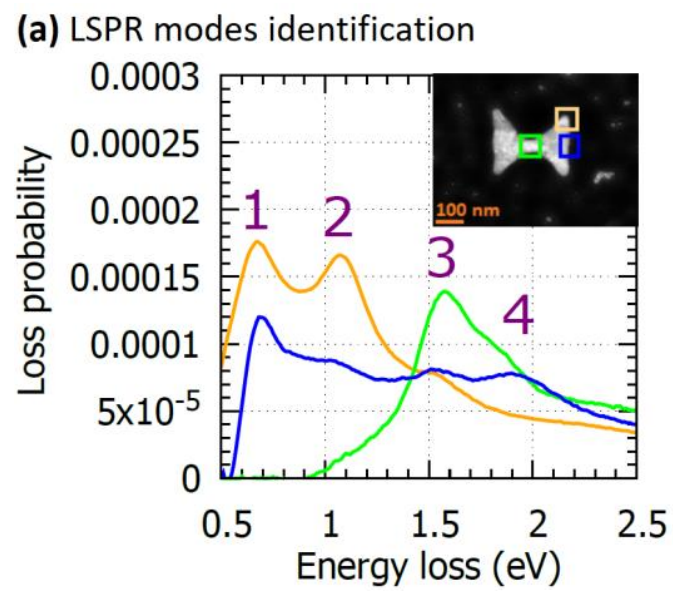
(b) measured loss probability maps at the energy of $0.69 \mathrm{eV}$ of particle and aperture
(c) simulated out-of-plane near field
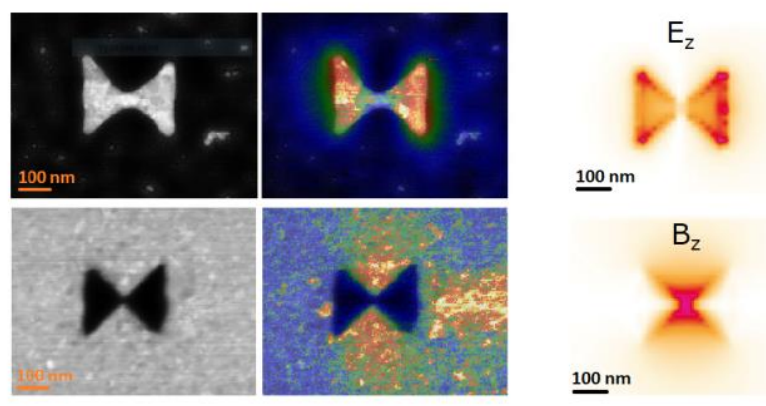

Figure 1. STEM-EELS mapping of the electric and magnetic near field in a diabolo antenna. (a) Identification of four LSPR modes in the diabolo antenna with the total length of $300 \mathrm{~nm}$. (b) STEM annular dark field micrographs of the diabolo (top) and inverted diabolo (bottom) antenna followed by measured loss probability maps at the energy loss of $0.69 \mathrm{eV}$ corresponding to the longitudinal dipole mode for both structures. (c) Calculated electric and magnetic out-of-plane near field distributions for the longitudinal dipole mode in the diabolo antenna. 

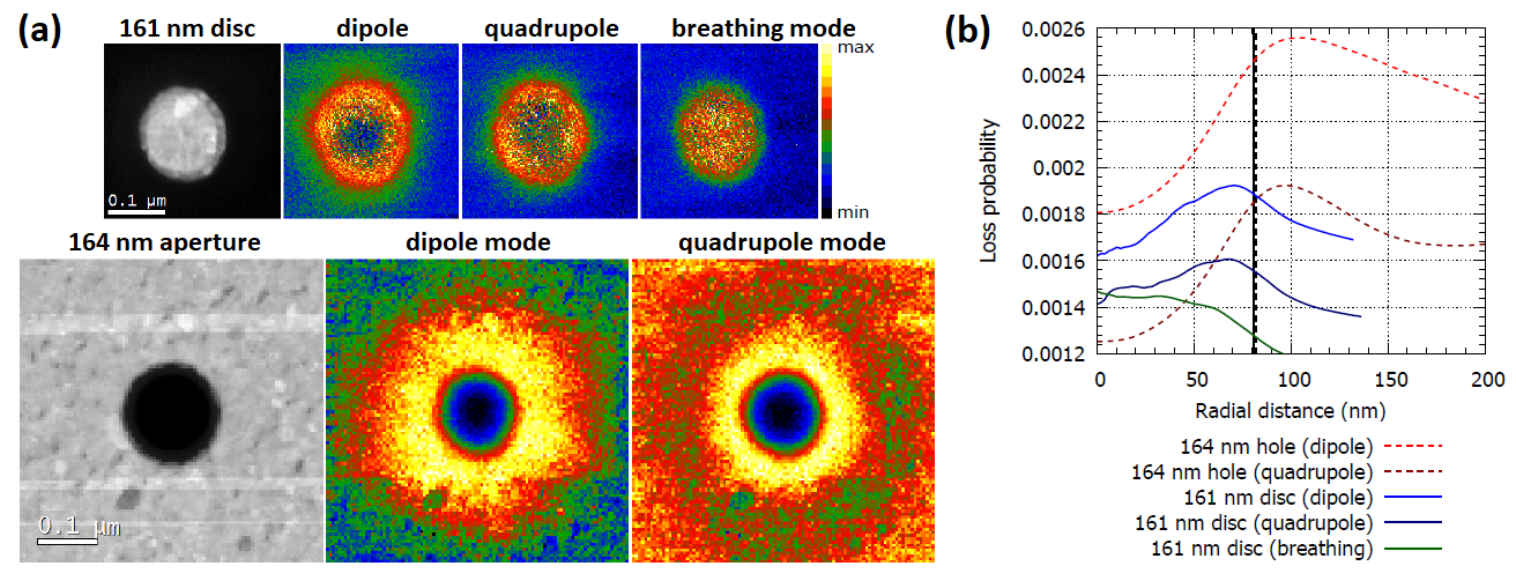

Figure 2. STEM-EELS mapping of the LSPR in a gold disc-shaped plasmonic particle and aperture. (a) STEM annular dark field micrograph of the particle (top) and the aperture (bottom) with measured loss probability maps showing the spatial distribution of LSPR modes: the dipole, quadrupole, and breathing mode. (b) Radial dependency of the loss probability for the LSPR modes.

\section{References}

[1] F. J. García de Abajo, Optical excitations in electron microscopy. Rev. Mod. Phys. 82 (2010) 209-275, doi: 10.1103/RevModPhys.82.209.

[2] M. Hentschel, T. Weiss, S. Bagheri, H. Giessen, Babinet to the half: Coupling of solid and inverse plasmonic structures, Nano Lett. 13 (2013) 4428-4433, doi: 10.1021/n1402269h.

[3] M. Horák, K. Bukvišová, V. Švarc, J. Jaskowiec, V. Křápek, T. Šikola, Comparative study of plasmonic antennas fabricated by electron beam and focused ion beam lithography, Sci. Rep. 8 (2018) 9640, doi: 10.1038/s41598-018-28037-1.

[4] V. Křápek, A. Konečná, M. Horák, F. Ligmajer, M. Stöger-Pollach, M. Hrtoň, J. Babocký, T. Šikola, Independent engineering of individual plasmon modes in plasmonic dimers with conductive and capacitive coupling, Nanophotonics 9 (2020) 623-632, doi: 10.1515/nanoph-2019-0326.

[5] M. Horák, V. Křápek, M. Hrtoň, A. Konečná, F. Ligmajer, M. Stöger-Pollach, T. Šamořil, A. Paták, Z. Edes, O. Metelka, J. Babocký, T. Šikola, Limits of Babinet's principle for solid and hollow plasmonic antennas, Sci. Rep. 9 (2019) 4004, doi: 10.1038/s41598-019-40500-1.

[6] Supported by Czech Science Foundation (17-25799S), European Union's Horizon 2020 program under the project SINNCE (810626), MEYS CR under the projects CzechNanoLab (LM2018110, 2020-2022) supporting the CEITEC Nano Research Infrastructure and CEITEC 2020 (LQ1601), and Brno University of Technology (FSI-S-17-4482, FSI/STI-J-17-4623, and FSI/STI-J-18-5225). M. H. acknowledges the support of Thermo Fisher Scientific and CSMS scholarship 2019. 